\title{
NOTES ON A CASE OF VOLVULUS OF THE CACUM, SECONDARY TO MALIGNANT DISEASE OF THE SIGMOID FLEXURE OF THE COLON.
}

\author{
BY JOHN LEO KEEGAN, F.R.C.S.; \\ Surgeon to Jervis Street Hospital.
}

[Read in the Section of Surgery, May 12, 1905.]

I venture to bring before you the following notes on a case of volvulus of the cæcum, which is the first of its kind that I have met in surgical practice.

J. K., aged fifty, labourer, was admitted to Jervis Street Hospital on December 1, 1904. He had fallen into the hold of a vessel in which he was employed, and had fractured a humerus, both bones of a forearm, and five ribs. He went on well until the morning of December 23, when he complained of griping colicky pains in the lower part of the abdomen and the flanks. The abdomen was much distended, resonant on percussion, and the liver dulness had almost disappeared. His appearance, however, was good; his pulse was strong, and only 72 per minute. At his bedside were two vessels filled with an inky-coloured, soursmelling fluid, which he had vomited without any effort on his part. His condition suggested some form of intestinal obstruction, and, as I noted that his bowels had not acted for two days, I ordered him castor oil and a turpentine enema, followed by one of soap and water, with the result that the bowels acted, and the pain and swelling rapidly disappeared.

On questioning him he told me that for several years past he had had occasionally similar attacks of pain, which a dose of Epsom salts relieved. He remained well until New Year's Eve, when the symptoms reappeared. I did not see him until the following evening, when I found no vomiting, pain not so intense as before, distention marked, pulse and temperature normal. I treated him as before, but with no result, and then, seeing that the distention was increasing, I advised operation, but to 
this his wife would not consent. On the next day his distention became more marked, his temperature was $100^{\circ} \mathrm{F}$., pulse 90 , and he had slight attacks of vomiting, with distressing hiccough.

On the morning of the third day I asked my colleague, Mr. Kennedy, to see him with me, but, although it was evident that death would soon ensue without operation, it was not until the evening of the third day that consent for this procedure was obtained. That night, being kindly assisted by Mr. Kennedy, I opened the abdomen in the middle line below the umbilicus. On getting through the peritoneum, a quantity of foul gas escaped, there was evidence of peritonitis, and the intestines were deeply congested; but presenting into the wound was an enormously distended portion of bowel, mottled in places by gangrenous patches, which I found to be the cæcum. The distention seemed to end just below the hepatic flexure of the colon, and the remain. ing parts of the colon were apparently normal, as were also the coils of small intestine above. It was clearly a case of volvulus, so I brought the distended bowel as far out of the abdomen as possible, then incised it, and allowed a large quantity of foul gas and focal fluid to escape. Seeing, however, that the bowel was gangrenous, I resected the entire mass, after clamping the bowel above and below; I was also obliged to remove portion of the mesentry, the vessels of which were thrombosed. I next washed out the abdomen with hot saline solution, and, as the patient's condition was one of extreme gravity, I simply brought out both ends of the bowel, sutured them to the abdominal wall, inserted Paul's tubes, and washed out the intestine. That night he seemed fairly well, the temperature was $99^{\circ} \mathrm{F}$., pulse 120 ; drainage was going on satisfactorily, so he was given a hypodermic of strychnin and morphia, with hot water to sip. He was quiet during the night, and next morning seemed to be holding his own; he was free from pain, and remained so during the day, but in the evening there were some vomiting and hiccough, which continued during the night. On the following day he was weaker, and, lapsing into a semi-comatose state, he died at $7 \mathrm{p} . \mathrm{m}$., having lived for two days after the operation.

The post-mortem examination revealed signs of general peritonitis; the bowel at the site of the wound was healthy, and had drained well, but there was a hard mass just at the brim of the pelvis on the lelt side, which on removal I found to be cancerous. 
This caused an absolute obliteration of the lumen of the bowel, as water would not pass through it at this point.

I received the following report from Professor McWeeney, Bacteriologist to the Hospital :-

"Diameter of bowel above stricture, $5 \frac{1}{2}$ inches; below, 2 inches. Mucous membrane above stricture is smooth and devoid of rugæ, that below is very rugose.

"Length of stricture, 2 inches ; it is so tight that I can hardly push a fine pen-handle through it.

"The greater purt of the cancerous tissue is near lower part. The upper part 'tails off' into a sort of collar, which projects into the lumen of the bowel after the manner of a cervix uteri; its microscopic structure is an adeno-carcinoma."

The points of interest in this case are:-

1. The fact that the patient showed no signs of having malignant disease until a few days before his death, although he had a cancerous growth which almost occluded the bowel.

2. That the volvulus was limited to the cæcum, and left: the remaining portion of the large intestine free-the dilatation mentioned in Professor MCWeeney's report being only immediately above the stricture.

3. The enormous size of the cæcum, which seemed to fill the entire abdominal carity. 